\title{
COMPACT HERMITIAN SURFACES OF CONSTANT ANTIHOLOMORPHIC SECTIONAL CURVATURES
}

\author{
VESTISLAV APOSTOLOV, GEORGI GANCHEV, AND STEFAN IVANOV \\ (Communicated by Christopher Croke)
}

\begin{abstract}
Compact Hermitian surfaces of constant antiholomorphic sectional curvatures with respect to the Riemannian curvature tensor and with respect to the Hermitian curvature tensor are considered. It is proved: a compact Hermitian surface of constant antiholomorphic Riemannian sectional curvatures is a self-dual Kaehler surface; a compact Hermitian surface of constant antiholomorphic Hermitian sectional curvatures is either a Kaehler surface of constant (non-zero) holomorphic sectional curvatures or a conformally flat Hermitian surface.
\end{abstract}

\section{INTRODUCTION}

The classification of the compact oriented self-dual Einstein Riemannian 4manifolds is an interesting problem completely solved when the scalar curvature is non-negative (see [13], [14]), but it is still open when the scalar curvature is negative. In the complex case, compact Einstein self-dual Hermitian surfaces are described by C. Boyer in [6].

On a self-dual Hermitian 4-manifold $(\mathbf{M}, J, g)$ the vanishing of the $J$-invariant part of the traceless Ricci tensor is equivalent to the sectional curvatures being pointwise constant on the holomorphic 2-planes (see [16], [1]). Thus, in the complex case the notion of pointwise constant holomorphic sectional curvatures can be considered as a generalization of the Einstein self-dual surfaces. Unfortunately there are no compact non-Kaehler examples according to the recent result in [1] (in some particular cases see also [17], [18]).

In this note we consider self-dual Hermitian surfaces with vanishing $J$-antiinvariant part of the Ricci tensor or equivalently, self-dual surfaces with $J$-invariant Ricci tensor. We show that these conditions are equivalent to the sectional curvatures being pointwise constant on the antiholomorphic (or totally real, or Lagrangian) 2-planes and construct a compact non-Kaehler example. Thus, a Hermitian surface of pointwise constant antiholomorphic sectional curvatures can be

Received by the editors March 22, 1995 and, in revised form, July 28, 1996.

1991 Mathematics Subject Classification. Primary 53C15, 53C55, 53B35.

Key words and phrases. Compact Hermitian surfaces, antiholomorphic Riemannian and antiholomorphic Hermitian sectional curvatures, self-dual Hermitian surfaces.

The first author was supported by Contract MM 423/1994 with the Ministry of Science and Education of Bulgaria; the second author was supported by Contract MM 413/1994 with the Ministry of Science and Education of Bulgaria; and the third author was supported by Contract MM 413/1994 with the Ministry of Science and Education of Bulgaria and by Contract 219/1994 with the University of Sofia "St. Kl. Ohridski". 
considered as a non-trivial generalization of the Einstein self-dual Hermitian surfaces even in the compact case.

We study compact Hermitian surfaces of constant antiholomorphic sectional curvatures which are "a priori" a natural extension of the class of compact Kaehler self-dual surfaces described in [4], [7], [15]. We prove the following classification result

Theorem 1.1. Any compact Hermitian surface of constant antiholomorphic Riemannian sectional curvatures is a self-dual Kaehler surface; i.e. it is biholomorphically isometric to one of the following:

i) the complex projective space $\mathbf{C P}^{2}$ with the Fubini-Study metric;

ii) a compact quotient of the unit ball in $\mathbf{C}^{2}$ with the Bergman metric;

iii) a complex torus with its flat Kaehler metric;

iv) a hyper elliptic surface with its flat Kaehler metric;

v) a $\mathbf{C P}^{1}$-bundle over a Riemannian surface $\Sigma_{g}$ of genus $g \geq 2$ with the conformally flat Kaehler metric which locally is the product of the +1-curvature metric on $\mathbf{C P}^{1}$ and-1-curvature metric on $\Sigma_{g}$.

Similar questions are studied with respect to the Hermitian connection. In the compact case the pointwise constancy of the holomorphic sectional curvatures leads to the metric being Kaehler ([3], [1]). By contrast, the condition of pointwise constancy of the Hermitian sectional curvatures on the aniholomorphic 2-planes is conformally invariant, which is somewhat surprising. We give a complete description of the compact Hermitian surfaces with pointwise constant antiholomorphic Hermitian sectional curvature with the following:

Theorem 1.2. A Hermitian surface is of pointwise constant antiholomorphic sectional curvatures with respect to the Hermitian connection if and only if it is selfdual. If moreover it is compact and the antiholomorphic sectional curvatures are constant then it is either a Kaehler surface of constant (non-zero) holomorphic sectional curvatures, or a conformally flat Hermitian surface.

\section{Preliminaries}

Let $(\mathbf{M}, J, g)$ be a Hermitian surface with complex structure $J$ and compatible Riemannian metric $g$. The algebra of all $C^{\infty}$ vector fields on $\mathbf{M}$ will be denoted by XM. The Kaehler form $\Omega$ on $\mathbf{M}$ is defined by $\Omega(X, Y)=g(X, J Y) ; X, Y \in \mathbf{X M}$. With respect to local holomorphic coordinates $\left\{z^{\alpha}\right\}(\alpha=1,2)$, we have $\Omega_{\alpha \bar{\beta}}=$ $-\sqrt{-1} g_{\alpha \bar{\beta}}$. The Lee form $\omega$ of the Hermitian structure is defined by $\omega=\delta \Omega \circ J$ or in local holomorphic coordinates $\omega_{\alpha}=\sqrt{-1} g^{\beta \bar{\gamma}} d \Omega_{\alpha \beta \bar{\gamma}}$ (summation convention is assumed). It is well known that $d \Omega=\omega \wedge \Omega$.

We shall consider two connections on $\mathbf{M}$.

The Levi-Civita connection will be denoted by $\nabla$ and the curvature tensor $R$ of type $(1,3)$ is defined by: $R=[\nabla, \nabla]-\nabla_{[,]}$. (We shall denote the curvature tensor of type $(0,4)$ by the same letter.) Further $\rho$ and $\tau$ will stand for the Ricci tensor and the scalar curvature, respectively. The $*$-Ricci tensor $\rho^{*}$ and the corresponding $*$-scalar curvature $\tau^{*}$ are determined by the equalities:

$$
\rho^{*}(X, Y)=\sum_{j=1}^{4} R\left(e_{j}, X, J Y, J e_{j}\right)=-\frac{1}{2} \sum_{j=1}^{4} R\left(X, J Y, e_{j}, J e_{j}\right), \quad \tau^{*}=\operatorname{tr}\left(\rho^{*}\right)
$$

where $e_{1}, e_{2}, e_{3}, e_{4}$ is an orthonormal local basis on $\mathbf{M}$. 
It is well known that (cf. [12], [21])

$$
\tau-\tau^{*}=\|\omega\|^{2}+2 \delta \omega .
$$

The Hermitian connection (or the Chern connection) will be denoted by $D$ and $K$ will stand for its curvature tensor.

The relation between the Levi-Civita connection and the Hermitian connection is given by:

$$
g\left(D_{X} Y, Z\right)=g\left(\nabla_{X} Y, Z\right)+\frac{1}{2} d \Omega(J X, Y, Z) ; X, Y, Z \in \mathbf{X M}
$$

Hence, the Hermitian curvature $K$ and the Riemannian curvature $R$ on a Hermitian surface are related by [21]:

$$
\begin{gathered}
2 K(X, Y, Z, U)=2 R(X, Y, Z, U)+L(X, U) g(Y, Z) \\
+L(Y, Z) g(X, U)-L(Y, U) g(X, Z)-L(X, Z) g(Y, U) \\
+\frac{\|\omega\|^{2}}{2}[g(X, Z) g(Y, U)-g(Y, Z) g(X, U)]+\Omega(Z, U) d(\omega \circ J)(X, Y),
\end{gathered}
$$

where $L(X, Y)=\left(\nabla_{X} \omega\right) Y+\frac{1}{2} \omega(X) \omega(Y)$.

The Hermitian scalar curvatures $u$ and $v$ are defined by:

$$
u=-\frac{1}{4} \sum_{i=1}^{4} \sum_{j=1}^{4} K\left(e_{i}, J e_{i}, e_{j}, J e_{j}\right) ; \quad v=\frac{1}{2} \sum_{i=1}^{4} \sum_{j=1}^{4} K\left(e_{i}, e_{j}, e_{j}, e_{i}\right),
$$

where $e_{1}, e_{2}, e_{3}, e_{4}$ is an orthonormal local basis on $\mathbf{M}$. By using (2.2) it is easily checked that the scalar curvatures $\tau, \tau^{*}, u$ and $v$ are related by the following well known formulas (cf. [12], [21])

$$
4 u=\tau+\tau^{*}+2\|\omega\|^{2} ; \quad 4 v=2 \tau^{*}+\|\omega\|^{2} .
$$

Note also that the conformal scalar curvature $\kappa$ is given by (cf. [12], [21])

$$
\kappa=\frac{3 \tau^{*}-\tau}{2}=4 v-2 u
$$

As an immediate consequence of $(2.2)$ we obtain that the $J$-antiinvariant part of the Ricci tensor is given by (cf. [21])

$$
\begin{aligned}
\rho(X, Y)-\rho(J X, J Y)=-\frac{1}{2} & {\left[\left(\nabla_{X} \omega\right) Y+\left(\nabla_{Y} \omega\right) X+\omega(X) \omega(Y)\right.} \\
& \left.-\left(\nabla_{J X} \omega\right) J Y-\left(\nabla_{J Y} \omega\right) J X-\omega(J X) \omega(J Y)\right] .
\end{aligned}
$$

The Riemannian metric $g$ induces a metric on the bundle $\Lambda^{2}$ of the 2-vectors on $\mathbf{M}$ by the equality $g\left(X_{1} \wedge X_{2}, X_{3} \wedge X_{4}\right)=\operatorname{det}\left(g\left(X_{i}, X_{j}\right)\right)$. The curvature operator $\mathcal{R}$ can be considered as the symmetric endomorphism of $\Lambda^{2}$ defined by $g(\mathcal{R}(X \wedge Y),(Z \wedge W))=-R(X, Y, Z, W)$. The Hodge star operator defines an endomorphism $*: \Lambda^{2} \rightarrow \Lambda^{2}$ with $*^{2}=\mathrm{Id}$. Set $\mathcal{B}=\frac{1}{2}(\mathcal{R}-* \mathcal{R} *), \mathcal{W}=\frac{1}{2}(\mathcal{R}+* \mathcal{R} *)-\frac{\tau}{12} I d$, $\mathcal{W}_{+}=\frac{1}{2}(\mathcal{W}+* \mathcal{W}), \mathcal{W}_{-}=\frac{1}{2}(\mathcal{W}-* \mathcal{W}) ;$ then

$$
\mathcal{R}=\frac{\tau}{12} I d+\mathcal{B}+\mathcal{W}_{+}+\mathcal{W}_{-}
$$

is the irreducible decomposition of $\mathcal{R}$ under the action of $\mathrm{SO}(4)$ found by Singer and Thorpe $([19])$. Note that $\mathcal{B}$ and $\mathcal{W}$ represent the traceless Ricci tensor and 
the Weyl conformal tensor respectively. We recall that the manifold is said to be self-dual (anti-self-dual) if $\mathcal{W}_{-}=0$ (resp. $\left.\mathcal{W}_{+}=0\right)$

Denote by $\chi, p_{1}$ and $c_{1}^{2}$ the Euler characteristic, the first Pontrjagin number and the square of the first Chern class of $\mathbf{M}$, respectively. According to the Chern-Weil theory we have:

$$
\begin{gathered}
\chi=\frac{1}{8 \pi^{2}} \int_{\mathbf{M}}\left[\left\|\mathcal{W}_{+}\right\|^{2}+\left\|\mathcal{W}_{-}\right\|^{2}+\frac{\tau^{2}}{24}-\|\mathcal{B}\|^{2}\right] d V \\
p_{1}=\frac{1}{4 \pi^{2}} \int_{\mathbf{M}}\left[\left\|\mathcal{W}_{+}\right\|^{2}-\left\|\mathcal{W}_{-}\right\|^{2}\right] d V
\end{gathered}
$$

Hence, since $c_{1}^{2}=2 \chi+p_{1}$ (cf. [22]), we get

$$
c_{1}^{2}=\frac{1}{4 \pi^{2}} \int_{\mathbf{M}}\left[2\left\|\mathcal{W}_{+}\right\|^{2}+\frac{\tau^{2}}{24}-\|\mathcal{B}\|^{2}\right] d V
$$

Moreover, any compact Hermitian surface satisfies (cf. [5], [16])

$$
\int_{\mathbf{M}}\left\|\mathcal{W}_{+}\right\|^{2} d V=\frac{1}{8} \int_{\mathbf{M}}\left[\|d \omega\|^{2}+\frac{\kappa^{2}}{3}\right] d V
$$

(Here and henceforth $\|\cdot\|^{2}$ denotes the tensor norm on $\Lambda^{2}$ ).

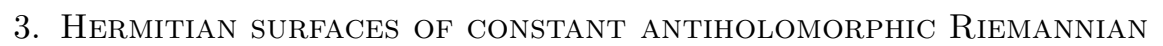 SECTIONAL CURVATURES}

Let $(\mathbf{M}, J, g)$ be a $2 n$-dimensional almost Hermitian manifold. A 2-plane $E$ in the tangent space $T_{p} \mathbf{M}$ at a point $p \in \mathbf{M}$ is said to be antiholomorphic (or totally real) if $J E \perp E$, where the action of $J$ on a 2-plane, or equivalently on a 2-vector $X \wedge Y$, is defined by $J(X \wedge Y)=J X \wedge J Y$.

An almost Hermitian manifold $(\mathbf{M}, J, g)$ is said to be of pointwise constant antiholomorphic Riemannian sectional curvatures $\mu(p)$ if

$$
\frac{R(X, Y, Y, X)}{g(X, X) g(Y, Y)-g^{2}(X, Y)}=\mu(p)
$$

whenever $E=\operatorname{span}\{X, Y\}$ is an antiholomorphic 2-plane in $T_{p} \mathbf{M}, p \in \mathbf{M}$.

Almost Hermitian manifolds of pointwise constant antiholomorphic sectional curvatures have been studied in [9], [8].

When $(M, J, g)$ is a 4-dimensional Hermitian manifold an observation of the referee leads to the following simple characterisation of the antiholomphic 2-planes: a 2-plane $E_{p}=\operatorname{span}\{X, Y\}$ of $T_{p} \mathbf{M}, p \in \mathbf{M}$, is antiholomorphic (or totally real) if and only if the corresponding 2 -vector $\alpha_{p}=X \wedge Y$ is orthogonal to the Kaehler form $\Omega$, i.e. $\alpha_{p}$ is Lagrangian. This is also equivalent to the self-dual part $\alpha_{+}$of $\alpha$ being $J$-antiinvariant.

We start with the following local description of the Hermitian surfaces of pointwise constant antiholomorphic sectional curvatures

Proposition 3.1. A Hermitian surface $(\mathbf{M}, J, g)$ is of pointwise constant antiholomorphic Riemannian sectional curvature if and only if it is self-dual and has $J$ invariant Ricci tensor, i.e. $\mathcal{W}_{-}=0$ and $\rho(J, J)=\rho($,$) .$

The antiholomorphic sectional curvature $\mu(p)$ is given by

$$
\mu=\frac{5 \tau-3 \tau^{*}}{48} \text {. }
$$


Proof. The statement can be proved applying considerations in [9]. Here we give a direct proof.

The Hermitian stricture $J$ induces an additional splitting of the components of the curvature operator in (2.6) as follows [20]:

a) The operator $\mathcal{B}$ splits into a sum of its $J$-invariant part $\mathcal{B}_{1}$ and its $J$-antiinvariant part $\mathcal{B}_{2}$, corresponding to the splitting of the traceless Ricci tensor to the $J$-invariant and to the $J$-antiinvariant parts.

b) The positive Weyl tensor $\mathcal{W}_{+}$splits into the following two parts:

$$
\mathcal{W}_{+}^{1}=\operatorname{const}\left(I d-\frac{3}{2} \Omega \otimes \Omega\right), \quad \mathcal{W}_{+}^{2}=\frac{1}{2}\left(\mathcal{W}_{+}+J \circ \mathcal{W}_{+} \circ J\right) .
$$

Let $(\mathbf{M}, J, g)$ be a Hermitian surface of pointwise constant antiholomorphic sectional curvatures and $\alpha=X \wedge Y$ be an antiholomorphic 2-plane of unit length, i.e. $\|\alpha\|^{2}=g(X \wedge Y, X \wedge Y)=1$. Then the 2-planes $J \alpha$ and $\bar{\alpha}=J X \wedge Y$ are also antiholomorphic and of unit length. The condition of the proposition implies $R(X, Y, Y, X)=R(J X, J Y, J Y, J X)$ for any tangent vectors $X, Y$. This equality implies that the Ricci tensor is $J$-invariant or equivalently $\mathcal{B}_{2}=0$. Now (2.6) and (3.10) give

$$
\mathcal{R}(\alpha, \alpha)=\frac{\tau}{12}+\mathcal{W}_{+}(\alpha, \alpha)+\mathcal{W}_{-}(\alpha, \alpha)
$$

and $\mathcal{W}_{+}(\alpha, \alpha)=\mathcal{W}_{+}(\bar{\alpha}, \bar{\alpha})$. Since $\operatorname{tr} \mathcal{W}_{+}=0$ we have $\mathcal{W}_{+}(\alpha, \alpha)+\mathcal{W}_{+}(\bar{\alpha}, \bar{\alpha})+$ $\frac{1}{4} \mathcal{W}_{+}(\Omega, \Omega)=0$. On the other hand from $(2.6)$ we get: $\kappa=3 \mathcal{W}_{+}(\Omega, \Omega)$. The last three equalities imply

$$
\mathcal{W}_{+}(\alpha, \alpha)=-\frac{\kappa}{24},
$$

i.e. $\mathcal{W}_{+}(\alpha, \alpha)$ does not depend on $\alpha$. From (3.11) we obtain that $\mathcal{R}(\alpha, \alpha)$ does not depend on $\alpha$ if and only if $\mathcal{W}_{-}(\alpha, \alpha)$ does not depend on $\alpha$. Since $\operatorname{tr} \mathcal{W}_{-}=0$, the last conclusion implies that $\mathcal{W}_{-}$does not depend on any unit holomorphic 2-plane determined by $X, J X$. Since $J \mathcal{W}_{-}=\mathcal{W}_{-}$, i.e $\mathcal{W}_{-}$has the Keahlerian property, then $\mathcal{W}_{-}$is completely determined by its trace. So $\mathcal{W}_{-}=0$. Finally, from (3.11) and (3.12) we get $\mathcal{R}(\alpha, \alpha)=\mu=\frac{5 \tau-3 \tau^{*}}{48}$ which completes the proof. $\quad$ Q.E.D.

Further we shall use the following classification theorem for compact self-dual surfaces

Theorem 3.2 ([1]). Any compact self-dual Hermitian surface is conformally equivalent to one of the following:

i) the complex projective space $\mathbf{C P}^{2}$ with the Fubini-Study metric;

ii) a compact quotient of the unit ball in $\mathbf{C}^{2}$ with the Bergman metric;

iii a complex torus with its flat Kaehler metric;

iv) a hyper elliptic surface with its flat Kaehler metric;

v) a $\mathbf{C P}^{1}$-bundle over a Riemannian surface $\Sigma_{g}$ of genus $g \geq 2$ with the conformally flat Kaehler metric which locally is the product of the +1-curvature metric on $\mathbf{C P}^{1}$ and-1-curvature metric on $\Sigma_{g}$;

vi) a Hopf surface with its locally conformally Kaehler-flat metric of constant positive scalar curvature. 
From this theorem and (2.5) immediately follows

Corollary 3.3. A compact Hermitian surface $(\mathbf{M}, J, g)$ is of pointwise constant antiholomorphic sectional curvatures if and only if it is self-dual and

$$
\left(\nabla_{X} \omega\right) Y-\left(\nabla_{J X} \omega\right) J Y+\frac{1}{2}[\omega(X) \omega(Y)-\omega(J X) \omega(J Y)]=0 .
$$

Remark 3.4. Note that according to Proposition 3.1 and the well known classification of the compact self dual Kaehler surfaces any compact self-dual Kaehler surface is of constant antiholomorphic sectional curvature $\mu=\frac{\tau}{24}$.

Proof of Theorem 1.1. We shall start with the following lemmas of technical nature:

Lemma 3.5. Let $(\mathbf{M}, J, g)$ be a compact locally conformally Kaehler surface with $J$-invariant Ricci tensor. Then we have:

$$
\int_{\mathbf{M}} \rho(Q, Q) d V=\int_{\mathbf{M}}\left[\frac{\left(\tau-\tau^{*}\right)^{2}}{8}+\frac{\tau-\tau^{*}}{4}\|\omega\|^{2}\right] d V
$$

where $Q$ is the vector field dual to the Lee form $\omega$.

Proof of Lemma 3.5. Applying Weitzenboeck's formula to the 1-forms $\omega$ and $\theta=$ $\delta \Omega$ we have

$$
\begin{gathered}
\int_{\mathbf{M}} \frac{1}{2}\|d \theta\|^{2} d V=\int_{\mathbf{M}}\left[\rho(J Q, J Q)+\|\nabla \theta\|^{2}\right] d V, \\
\int_{\mathbf{M}}(\delta \omega)^{2} d V=\int_{\mathbf{M}}\left[\rho(Q, Q)+\|\nabla \omega\|^{2}\right] d V .
\end{gathered}
$$

By using the formula

$$
\left(\nabla_{X} \theta\right) Y=-\left(\nabla_{X} \omega\right) J Y+\frac{1}{2}\|\omega\|^{2} \Omega(X, Y)+\frac{1}{2}(\omega \wedge \theta)(X, Y)
$$

as well as Corollary 3.3 we calculate

$$
\begin{gathered}
\int_{\mathbf{M}}\|\nabla \theta\|^{2} d V=\int_{\mathbf{M}}\left[\|\nabla \omega\|^{2}+2 \delta \omega\|\omega\|^{2}+\|\omega\|^{4}\right] d V, \\
\int_{\mathbf{M}} \frac{1}{2}\|d \theta\|^{2} d V=\int_{\mathbf{M}}\left[2\|\nabla \omega\|^{2}+4 \delta \omega\|\omega\|^{2}+\frac{7}{4}\|\omega\|^{4}\right] d V .
\end{gathered}
$$

Substituting (3.16) and (3.17) into (3.14) and taking into account (3.15) we prove the lemma.

Q.E.D.

Lemma 3.6. Let $(\mathbf{M}, J, g)$ be a compact Hermitian surface which is locally conformal to a Kaehler-Einstein metric. Then we have

$$
\int_{\mathbf{M}} \rho(Q, Q) d V=\int_{\mathbf{M}} \frac{\kappa}{4}\|\omega\|^{2} d V .
$$

Proof of Lemma 3.6. The canonical Weyl connection $\nabla^{\prime}$ on $(\mathbf{M}, J, g)$ is related to the Levi-Civita connection $\nabla$ by the equality (see [12], [21])

$$
\nabla_{X}^{\prime} Y=\nabla_{X} Y-\frac{1}{2} \omega(X) Y-\frac{1}{2} \omega(Y) X+\frac{1}{2} g(X, Y) Q .
$$

Since $\nabla^{\prime}$ preserves the conformal structure, it follows that the Ricci tensor $\rho^{\prime}$ of $\nabla^{\prime}$ is conformally invariant and the conditions of Lemma 3.6 imply $\rho^{\prime}=\frac{\kappa}{4} g$. Using the 
formula (see [12], [21])

$$
\rho^{\prime}(X, Y)=\rho(X, Y)+\left(\nabla_{X} \omega\right) Y+\frac{1}{2} \omega(X) \omega(Y)-\frac{\tau-\tau^{*}+\|\omega\|^{2}}{4} g(X, Y),
$$

we get (3.18).

Q.E.D.

Lemma 3.7. Let $(\mathbf{M}, J, g)$ be a compact Hermitian surface. If the metric $g$ is conformal to a Kaehler metric of constant scalar curvature, then

$$
\int_{\mathbf{M}} \kappa\left(\tau-\tau^{*}+\|\omega\|^{2}\right) d V=0 .
$$

If moreover the conformal scalar curvature $\kappa$ is a non-zero constant then the metric $g$ is a Kaehler metric.

Proof of Lemma 3.7. Let $h=e^{-\sigma} g$ be the Kaehler metric of constant scalar curvature $s$. Then we have

$$
e^{\sigma} s=\tau-3 \triangle \sigma-\frac{3}{2}\|\omega\|^{2}, \quad e^{\sigma} s=\tau^{*}-\triangle \sigma-\frac{1}{2}\|\omega\|^{2}, \quad e^{\sigma} s=\kappa,
$$

where $\triangle$ denotes the Laplacian of the metric $g$. From these equalities we get

$$
\begin{aligned}
\int_{\mathbf{M}} \kappa\left(\tau-\tau^{*}+\|\omega\|^{2}\right) d V & =\int_{\mathbf{M}} 2 s e^{-\sigma}\left(\triangle \sigma+\|d \sigma\|^{2}\right) d V \\
& =2 s \int_{\mathbf{M}} e^{-\sigma}\left(\triangle \sigma+\|d \sigma\|^{2}\right) d V=0 .
\end{aligned}
$$

If $\kappa=$ const $\neq 0$ then the third equality of (3.19) implies $e^{\sigma}=$ const $\neq 0$. Hence, $g$ is a Kaehler metric.

Q.E.D.

Now we are ready to give the proof of Theorem 1.1.

Let $(\mathbf{M}, J, g)$ be a compact Hermitian surface of constant antiholomorphic Riemannian sectional curvatures $\mu=\frac{5 \tau-3 \tau^{*}}{48}$.

I. The case $\mu \leq 0$.

1) Let $\mathcal{W}_{+}=0$. According to (2.9) we have $\tau=3 \tau^{*}$ and $\tau-\tau^{*}=8 \mu \leq 0$. Integrating (2.1) we obtain

$$
\int_{\mathbf{M}}\left(\|\omega\|^{2}-8 \mu\right) d V=0
$$

Hence, $\omega=0$ and $g$ is a Kaehler metric.

$2)$ Let $\mathcal{W}_{+} \neq 0$. According to Theorem $3.2(\mathbf{M}, J, g)$ is conformally equivalent to a surface of type i) or ii). Then $c_{1}^{2}=3 \chi$ and from $(2.7),(2.8)$ it follows that

$$
\begin{gathered}
\int_{\mathbf{M}}\left[64\|\mathcal{B}\|^{2}-\left(\tau-\tau^{*}\right)\left(\tau+3 \tau^{*}\right)\right] d V \\
=\int_{\mathbf{M}}\left[64\|\mathcal{B}\|^{2}+9\left(\tau-\tau^{*}\right)^{2}-96 \mu\|\omega\|^{2}\right] d V=0 .
\end{gathered}
$$

Hence, $\tau=\tau^{*}$ and $g$ is a Kaehler metric.

II. The case $\mu>0$.

Let $f_{o}$ be the eccentricity function of the Hermitian surface $(\mathbf{M}, J, g)$ introduced and studied in [10], [11]. The function $f_{o}$ is positive on $\mathbf{M}$ and the sign of $\int_{\mathbf{M}}(10 u-16 v) f_{o} d V$ is a conformal invariant [2]. Taking into account (2.4) we get

$$
\int_{\mathbf{M}}(20 u-16 v) f_{o} d V=\int_{\mathbf{M}}\left(48 \mu+6\|\omega\|^{2}\right) f_{o} d V>0 .
$$


1) Let $\mathcal{W}_{+}=0$. Then Theorem 3.2 and (3.21) imply that the Hermitian surface $(\mathbf{M}, J, g)$ is conformally equivalent to a Hopf surface with its locally conformally Kaehler-flat metric. Then Lemma 3.6 implies $\int_{\mathbf{M}} \rho(Q, Q) d V=0$. Applying Lemma 3.5 and (2.9) we find

$$
\int_{\mathbf{M}}\left[\left(\tau-\tau^{*}\right)^{2}+16 \mu\|\omega\|^{2}\right] d V=0 .
$$

Hence, $\tau=\tau^{*}$ and $g$ is a Kaehler metric, a contradiction.

$2)$ Let $\mathcal{W}_{+} \neq 0$. In this case Theorem 3.2 and (3.21) imply $(\mathbf{M}, J, g)$ is conformally equivalent to $\mathbf{C P}^{2}$ with the Fubini-Study metric. Then $c_{1}^{2}=3 \chi$ and from Lemma 3.5, Lemma 3.6 and Lemma 3.7 we obtain

$$
\int_{\mathbf{M}}\left[\left(\tau-\tau^{*}\right)^{2}+\left(3 \tau-5 \tau^{*}\right)\|\omega\|^{2}\right] d V=0
$$

Since $\mu=\frac{3 \tau-5 \tau^{*}}{48}$ is constant, from (2.1) we obtain:

$$
\int_{\mathbf{M}}\left(3 \tau^{*}-5 \tau\right)\left(\tau-\tau^{*}-\|\omega\|^{2}\right) d V=0 .
$$

From these equalities and Lemma 3.7 it follows easily that

$$
\begin{gathered}
\int_{\mathbf{M}} \tau\|\omega\|^{2} d V=\frac{3}{2} \int_{\mathbf{M}}\left(\tau-\tau^{*}\right)^{2} d V, \quad \int_{\mathbf{M}} \tau^{*}\|\omega\|^{2} d V=\frac{11}{10} \int_{\mathbf{M}}\left(\tau-\tau^{*}\right)^{2} d V, \\
\int_{\mathbf{M}} 48 \mu\|\omega\|^{2} d V=\frac{21}{5} \int_{\mathbf{M}}\left(\tau-\tau^{*}\right)^{2} d V
\end{gathered}
$$

Since $c_{1}^{2}=3 \chi$, then $(3.20)$ implies

$$
\left.\int_{\mathbf{M}}\left[64\|\mathcal{B}\|^{2}+\frac{3}{5}\left(\tau-\tau^{*}\right)^{2}\right)\right] d V=0
$$

Hence, $\tau=\tau^{*}$ and $g$ is a Kaehler metric.

Q.E.D.

In [1] it is proved that there are no compact Hermitian surfaces of pointwise constant holomorphic sectional curvatures other than Kaehler surfaces of constant holomorphic sectional curvatures. With the next example we show that Theorem 1.1 cannot be improved in this sense, i.e. there is a compact non-Kaehler Hermitian surface of pointwise constant but not constant antiholomorphic sectional curvatures.

Example 1. Let $\left(\mathbf{M}=\mathbf{C P}^{1} \times \Sigma_{g}, J, h\right)$ be the compact conformally flat Kaehler surface of type v) in Theorem 3.2. If $\left(z^{1}, z^{2}\right)$ are local holomorphic coordinates we shall use the following substitution: $f_{\alpha}:=\frac{\partial f}{\partial z^{\alpha}}$. Let $h^{\prime}=e^{f} h$ be a metric conformal to the metric $h$, so that $\left(\mathbf{M}, J, h^{\prime}\right)$ is of pointwise constant antiholomorphic sectional curvatures $\mu \neq$ const. According to Corollary 3.3 this condition is equivalent to the equality

$$
\nabla_{\alpha} f_{\beta}+\frac{1}{2} f_{\alpha} f_{\beta}=0
$$

with respect to the Levi-Civita connection $\nabla$ of the metric $h$.

Suppose $f: \mathbf{C P}^{1} \rightarrow \mathbf{R}$. If $p$ is an arbitrary point in $\mathbf{C P}^{1}$, consider stereographic coordinates $(x, y)$ in $\mathbf{C} \mathbf{P}^{1} \backslash\{p\}$. Then (3.22) is equivalent to the system

$$
f_{x x}-f_{y y}+\frac{1}{2}\left(f_{x}^{2}-f_{y}^{2}\right)+2 \frac{x f_{x}-y f_{y}}{1+x^{2}+y^{2}}=0,
$$




$$
2 f_{x y}+f_{x} f_{y}+2 \frac{x f_{y}+y f_{x}}{1+x^{2}+y^{2}}=0 .
$$

It is easy to check that the function $f=\frac{2}{1+\ln \left(1+x^{2}+y^{2}\right)} ; f(p)=0$ is a $\mathbf{C}^{\infty}$ solution of (3.23),(3.24) and (3.22). Hence, $\left(\mathbf{M}, J, e^{f} h\right)$ is a compact non-Kaehler Hermitian surface of pointwise constant antiholomorphic Riemannian sectional curvatures.

\section{Hermitian surfaces of CONStant antiholomorphic Hermitian SECTIONAL CURVATURES}

An almost Hermitian manifold $(\mathbf{M}, J, g)$ is said to be of pointwise constant antiholomorphic Hermitian sectional curvatures $\nu(p)$ if

$$
\frac{K(X, Y, Y, X)}{g(X, X) g(Y, Y)-g^{2}(X, Y)}=\nu(p),
$$

whenever $E=\operatorname{span}\{X, Y\}$ is an antiholomorphic 2-plane in $T_{p} \mathbf{M}, p \in \mathbf{M}$.

Proof of Theorem 1.2. Let $(\mathbf{M}, J, g)$ be a Hermitian surface of pointwise constant antiholomorphic Hermitian sectional curvatures. We define the following tensor

$$
\begin{gathered}
P(X, Y, Z, W)=\frac{1}{6}(2 K(X, Y, Z, W)+2 K(Z, W, X, Y) \\
-K(Y, Z, X, W)-K(X, W, Y, Z)-K(Z, X, Y, W)-K(Y, W, Z, X))
\end{gathered}
$$

which has the same antiholomorphic sectional curvatures as the Hermitian curvature tensor $K$. Using the well known symmetries of the Hermitian curvature $K$ it is easy to check that the tensor $P$ has the same symmetries as the Riemannian curvature tensor $R$. Moreover, the defining equality (4.25) of $P$ implies $P(J, J, J, J)=P(,,$,$) . Hence, the Ricci tensor of P$ is $J$-invariant. The same arguments as in Proposition 3.1 show that the tensor $P$ has pointwise constant antiholomorphic sectional curvatures $\nu(p)$ (and hence $K$ has the same property) if and only if the tensor $P$ is self-dual, i.e. the corresponding half Weyl tensor $\mathcal{W}_{-}^{P}$ of $P$ vanish. Using the relation (2.2) between the Riemannian curvature $R$ and the Hermitian curvature $K$ from (4.25) we get $\mathcal{W}_{-}^{P}=0=\mathcal{W}_{-}, \quad \nu=\frac{\kappa}{6}$ i.e. $(\mathbf{M}, J, g)$ is a self-dual Hermitian surface.

Now, let $(\mathbf{M}, J, g)$ be a Hermitian surface of constant antiholomorphic Hermitian sectional curvatures. Then $(\mathbf{M}, J, g)$ is a self-dual Hermitian surface with constant conformal scalar curvature $\kappa$. By Theorem $3.2 \mathrm{~g}$ is a locally conformal Kaehler metric.

$1)$. The case $\kappa=0$. The formula (2.9) implies that $(\mathbf{M}, J, g)$ is a locally conformal flat Hermitian surface.

$2)$. The case $\kappa \neq 0$. According to (2.9) the metric $g$ is not locally conformal flat. Then Theorem 3.2 implies that $(\mathbf{M}, J, g)$ is conformally equivalent to a Kaehler metric $g^{\prime}$ of constant holomorphic sectional curvatures. According to Lemma 3.7 the metric $g$ has to be a Kaehler metric of constant holomorphic sectional curvatures.

Q.E.D.

\section{ACKNOWLEDGEMENTS}

We would like to thank the referee for his remarks and helpful suggestions. 


\section{REFERENCES}

1. V.Apostolov, J.Davidov, O.Muskarov, Compact self-dual Hermitian surfaces., Trans. A.M.S. 348 (1996), 3051-3063. MR 96j:53040

2. A.Balas, Compact Hermitian manifolds of constant holomorphic sectional curvature., Math. Z. 189 (1985), 193-210. MR 86f:53072

3. A.Balas, P.Gauduchon, Any Hermitian metric of constant non-positive (Hermitian) holomorphic sectional curvature on a compact complex surface is Kähler., Math. Z. 190 (1985), 39-43. MR 86k:53066

4. J.P.Bourguignon, Les variétés de dimension 4 à signature non nulle dont la courbure est harmonique sont d'Einstein, Invent.Math. 63 (1981), 263-286. MR 82g:53051

5. Ch. Boyer, Conformal duality and compact complex surfaces., Math. Ann. 271 (1986), 517 - 526. MR 87i:53068

6. Ch. Boyer, Self-dual and anti-self-dual Hermitian metrics on compact complex surfaces, in J.A.Isenberg (Ed.) Mathematics and general relativity, Proceedings, Santa Cruz 1986 (Contemp. Math. 71), Providence AMS 1988, 105-114. MR 89h:53127

7. A.Derdzinski, Self-dual Kähler manifolds and Einstein manifolds of dimension four, Compositio Math. 49 (1983), 405-433. MR 84h:53060

8. M.Falcitelli, A.Farinola, Locally conformal Kähler manifolds with pointwise constant antiholomorphic sectional curvature., Riv. Mat. Univ. Parma (4) 17 (1991), 295-314. MR 93h:53070

9. G.Ganchev, On Bochner curvature tensors in almost Hermitian manifolds., Pliska Studia mathematica bulgarica 9 (1987), 33 - 43. MR 88e:53045

10. P.Gauduchon, Fibrés hermitiens à endomorphisme de Ricci non négatif., Bull. Soc. Math. France 105 (1977), 113-140. MR 58:6375

11. P.Gauduchon, Le théorème de l'excentricité nulle., C.R.Acad. Sci. Paris, Ser. A 285 (1977), 387-390. MR 57:10664

12. P.Gauduchon, La 1-forme de torsion d'une variété hermitienne compacte, Math.Ann. $\mathbf{2 6 7}$ (1984), 495-518. MR 87a:53101

13. N.J.Hitchin, Compact four-dimensional Einstein manifolds, J.Diff.Geom. 9 (1974), 435-441. MR 50:3149

14. N.J.Hitchin, Kählerian twistor spaces, Proc.London Math.Soc. 43 (1981), 133-150. MR 84b:32014

15. M. Itoh, Self-duality of Kähler surfaces, Compositio Math. 51 (1984), 265-273. MR 85m:53079

16. T.Koda, Self-dual and anti-self dual Hermitian surfaces., Kodai Math. J. 10 (1987), 335 342. MR 89a:53053

17. T.Sato, K.Sekigawa, Hermitian surfaces of constant holomorphic sectional curvature., Math. Z. 205 (1990), 659 - 668. MR 91m:53052

18. T.Sato, K.Sekigawa, Hermitian surfaces of constant holomorphic sectional curvature II., Tamkang J. Math. 23 N2 (1992), 137 - 143. MR 93g:53094

19. I.Singer, J.Thorpe, The curvature of 4-dimensional Einstein spaces., Papers in Honor of Kodaira, Univ. Tokyo Press, Tokyo, 1969, 335 - 365. MR 41:959

20. F.Tricerri, L.Vanhecke, Curvature tensors on almost Hermitian manifolds., Trans. Amer. Math. Soc. 267 (1981), 365 - 397. MR 82j:53071

21. I.Vaisman, Some curvature properties of complex surfaces., Ann. Math. Pura Appl. 132 (1982), 1 - 18. MR 84i:53064

22. W.Wu, Sur la structure presque complexe d'une variété différentiable réelle de dimension 4., C.R.Acad.Sci. Paris, 227 (1948), 1076 - 1078. MR 10:318b

(V. Apostolov and G. Ganchev) Bulgarian Academy of Science, Institute of Mathematics ACAD., G. Bonchev Str., Blok 8, 1113 Sofia Bulgaria

E-mail address, G. Ganchev: ganchev@math.acad.bg

(S. Ivanov) University of Sofia, Faculty of Mathematics and Informatics, Department of Geometry, bul. James Bouchier 5, 1164 Sofia, Bulgaria

E-mail address: ivanovsp@fmi.uni-sofia.bg 